\title{
Caractérisation phénotypique du néré (Parkia biglobosa (Jacq.) R. Br. ex G. Don, Fabaceae) dans trois types de savane de Côte d'Ivoire
}

\author{
Léonie Clémence KOUONON ${ }^{1 *}$, Kouamé Guillaume KOFFI ${ }^{1}$, Koffi ADJOUMANI ${ }^{2}$, \\ Anouman Désirée Sandrine $\mathrm{MOBIO}^{1}$ et Koudougnon Alice Estère GOBA ${ }^{1}$
}

\author{
${ }^{1}$ Université Nangui Abrogoua, Unité de Phytotechnie et Amélioration génétique, \\ 02 BP 801 Abidjan 02, Côte d'Ivoire. \\ ${ }^{2}$ Ecole Normale Supérieure d'Abidjan, 08 BP 10 Abidjan 08, Côte d'Ivoire. \\ *Auteur correspondant ; E-mail: lckouonon@yahoo.fr ; Tel: +225 47980064
}

\section{RESUME}

Afin de préserver les nombreux biens et services fournis par Parkia biglobosa (Jacq.) R. Br. ex G. Don, Fabaceae, il est fondamental de connaître la variabilité phénotypique de l'espèce pour sa domestication. L'objectif de cette étude est de déterminer des caractères morphologiques permettant d'identifier des arbresplus dans les savanes de Côte d'Ivoire. La variabilité phénotypique d'arbres distants d'au moins $100 \mathrm{~m}$ des trois types de savane a été étudiée à travers 22 caractères quantitatifs relatifs aux troncs, feuilles, fruits et graines. La matrice de données obtenue a été analysée avec différents tests tels que MANOVA suivie de ANOVA 1, AFD et la CAH. Les paramètres permettant de distinguer les arbres selon leur origine sont : hauteur totale d'arbre, épaisseur graine, poids de graine et pulpe par fruit, longueur du fruit, longueur et épaisseur du pédicelle, longueur et épaisseur du pédoncule. Les arbres en savane guinéenne ont de longs et épais pédoncules, des pédicelles épais et de longs fruits. Les arbres en savane sub-soudanaise sont hauts avec des graines et pédicelles épais, un poids de graine et de pulpe par fruit en moyenne élevé alors que les arbres en savane soudanaise sont hauts avec un long pédoncule.

(C) 2020 International Formulae Group. All rights reserved.

Mots clés: Parkia biglobosa, néré, variabilité phénotypique, conservation, savane.

\section{Phenotypical caracterization of african locust bean (Parkia biglobosa (Jacq.) R. Br. ex G. Don, Fabaceae) from three savannahs types in Côte d'Ivoire}

\begin{abstract}
To preserve goods and services provided by Parkia biglobosa (Jacq.) R. Br. ex G. Don, Fabaceae, it is essential to know the phenotypical variability of this species for domestication purpose. The objective of this study is to define morphological discriminant traits to identify tree-plus in the savannahs from Côte d'Ivoire. The phenotypical variability of trees distant at least $100 \mathrm{~m}$ from three types of savannah was studied through 22 quantitative traits related to trunks, leaves, fruits and seeds. Matrix data was analyzed with different tests such as MANOVA followed by ANOVA 1, AFD and CAH. Traits that allow distinguishing trees according to their origin are: total height, seed thickness, seed and pulp weight per fruit, fruit length, pedicel length and thickness, peduncle length and thickness. In the Guinean savannah, trees have long and thick peduncles, thick
\end{abstract}


pedicels and long fruits. In Sub-Sudanese savannah, trees are tall with thick seeds and pedicels, high seeds and pulp weight per fruit while Sudanese savannah trees are tall with a long peduncle.

(C) 2020 International Formulae Group. All rights reserved.

Keywords: Parkia biglobosa, locust bean, phenotypical variability, conservation, savannah.

\section{INTRODUCTION}

L'une des conséquences de la croissance démographique galopante est la dégradation rapide des écosystèmes naturels. Dès lors, les ressources naturelles disparaissent sous la pression des activités agricoles, minières et l'urbanisation car la destruction des écosystèmes s'accompagne de la perte des espèces qu'ils abritent. Pendant les trois premières décennies d'indépendance politique de la Côte d'Ivoire, sous l'impulsion de la course effrénée aux ressources, les zones forestières ont connu d'importantes dégradations à la faveur de la création des plantations industrielles de cacaoyers, caféiers, palmiers à huile et hévéas. Depuis les années 1990; avec la chute des prix des matières premières agricoles telles le café et le cacao, les activités se sont diversifiées et étendues dans les écosystèmes de savanes avec le développement des spéculations agricoles telles que l'anacarde et l'exploitation minière et des ligneux de savane. Par conséquent, on assiste à la destruction des ressources ligneuses de savane qui pourtant offrent des biens et services aux populations locales (Kouyaté, 2005). Il n'est pas rare de voir sur les marchés locaux, les produits dérivés des arbres de savanes prélevés dans les populations naturelles. Certes, ces produits contribuent indéniablement à l'amélioration des revenus des populations (Babalola, 2012 ; Sodique, 2016), mais ils accentuent la pression sur les arbres de savane qui ne bénéficient d'aucune mesure de protection (Kouyaté, 2005). Parmi les arbres de savane, Parkia biglobosa, communément appelé le néré, occupe une place importante au regard des valeurs que les populations locales lui confèrent. Il entre dans l'alimentation de plus de 20 millions de personnes réparties sur 20 pays africains (Shao, 2000) favorisant ainsi la réduction des déséquilibres nutritionnels. En effet, $100 \mathrm{~g}$ de moutarde africaine à base de graines de néré, renferment des quantités non négligeables de protides (36,5 $\mathrm{mg}$ ), lipides (28,8 g), fer (378 mg) (Camara et al., 2016), de vitamines B2, PP et de nombreux autres éléments nutritifs contenus dans ses graines (Olujobi, 2012; Modupe, 2014), ce qui garantit un pouvoir de diversification des éléments nutritifs. Le néré est utilisé, des racines à la cime, pour ses vertus médicinales et ses propriétés anti-diarrhéique, antimicrobienne, anti-hypertensive, antiinflammatoire, analgésique, antispasmodique, antihelminthique, antimalarial (Modupe, 2014). Les feuilles, les gousses, l'écorce et les racines de $P$. biglobosa contiendraient des alcaloïdes, tannins, saponines, flavonoïdes, stéroïdes, phénols, glycosides (Modupe, 2014; Dedehou et al., 2016). Parkia biglobosa est aussi une espèce fourragère fort appréciée par le bétail et un excellent fertilisant du sol (Koura et al., 2013 ${ }^{\mathrm{a}}$ ) et joue un rôle important dans sa protection contre l'érosion éolienne et pluviale (Bayala et al., 2005). On reconnait aussi d'autres biens et services écosystémiques majeurs de $P$. biglobosa dans la vie quotidienne des communautés locales : culturel, spirituel, énergie domestique, bois d'œuvre et sources de revenus (Babalola, 2012). Ces différents atouts ont emmené les populations de savane à se focaliser généralement sur leurs savoirs traditionnels et les interdits en vue de la sauvegarde de l'espèce. En effet, les populations de savane préservaient l'espèce même dans leurs exploitations agricoles pour ses nombreuses vertus (Lamien et al., 2011; Koura et al., 2013 ; Avana-Tchientcheu et al., 2019). Malgré cette forme de conservation traditionnelle, les arbres sont régulièrement coupés, notamment pour la confection de charbon de bois, entrainant une réduction drastique des peuplements. Aussi, les effets néfastes des changements climatiques, le faible taux de régénération (Ntui et al., 2012), 
le vieillissement des vergers, la propagation d'espèces exotiques envahissantes et la disparition des habitats naturels suscitent-ils des craintes pour la survie de l'espèce (HahnHadjali et Thiombiano, 2000 ; Teklehaimanot, 2004). Sur le long terme, ces pressions priveraient les populations locales de cette importante ressource. Il paraît donc indispensable de préserver cette espèce dans toute sa diversité à travers son aire de répartition en Côte d'Ivoire.

La diversité au sein d'une espèce peut s'apprécier à travers des paramètres morphologiques et moléculaires (Lassen et al., 2014 ; Ikabanga et al., 2017 ; AvanaTientcheu et al., 2019). Ainsi, des descripteurs morphologiques doivent-ils être identifiés pour une évaluation en routine de la variabilité phénotypique de l'espèce. En effet, les arbres forestiers sont caractérisés par une diversité génétique élevée donc un potentiel d'adaptation élevé (Kremer, 2000). L'aire de répartition de P. biglobosa est large et s'étend du Sénégal à l'ouest du continent, à l'Ouganda à l'est (Sina, 2006). Cette vaste aire de distribution et l'organisation en populations de très grande taille est un avantage pour un niveau de diversité génétique élevé (Amusa et al., 2014; Ikabanga et al., 2017). Aussi, les données morphologiques d'origine géographique ontelles contribué aux premières études de diversité génétique chez les arbres (Kouyaté et al., 2011; Koura et al., 2013 ${ }^{\text {b }}$ ). La grande diversité est liée à l'origine géographique et la différence entre individus d'une même population (Goba et al., 2019). Par conséquent, tout programme de domestication d'arbres devait s'appuyer sur le potentiel adaptatif qui se cache sous le vocable diversité génétique.

Dans le contexte actuel du réchauffement climatique et de la crise de la biodiversité, il faut éviter que le nombre de plantes ainsi que leur réservoir génétique diminuent. La recherche orientée vers la sauvegarde de la biodiversité s'avère d'une importance capitale. La domestication et la valorisation des ressources menacées dans des programmes de reboisement pourraient être une des voix les plus prometteuses pour assurer le plein succès du défi de sauvegarde de l'environnement. L'objectif de cette étude est de déterminer des caractères morphologiques discriminants pour identifier des arbres-plus de $P$. biglobosa dans les trois types de savane de Côte d'Ivoire.

\section{MATERIEL ET METHODES Sites d'étude}

Selon Guillaumet et Adjanohoun (1971) et Diarassouba et al. (2008), la végétation du Nord de la Côte d'Ivoire à partir du $8^{\text {ème }}$ parallèle est constituée de savane guinéenne, savane sub-soudanaise et soudanaise (Figure 1). Les peuplements de néré de ces trois types de savane sont l'objet de cette étude. Les mesures ont été prises sur des arbres dans quatre sites (Figure 1). Le site de Bouna qui est caractérisé par un climat de type soudanais connait deux saisons où la pluviométrie moyenne annuelle est de 1100 $\mathrm{mm}$ et une végétation de savanes boisée, arbustive et herbeuse avec des galeries forestières le long des cours d'eau. Le site de Bocanda, situé dans une région de transition climatique entre le climat subtropical et le climat tropical humide, quant à lui est caractérisé par une végétation de type guinéenne et un climat de type subtropical à quatre saisons (deux saisons sèches alternant avec deux saisons des pluies), la pluviométrie annuelle moyenne est de $1500 \mathrm{~mm}$. Enfin, les sites de Koro et Kahanso sont caractérisés par un climat de type soudano-guinéen à deux saisons avec $1300 \mathrm{~mm}$ de pluie par an et une végétation de types savanes arborée, boisée et arbustive selon le niveau de pression agricole.

\section{Méthodes \\ Evaluation des paramètres}

Dans chaque peuplement, des arbres de Parkia biglobosa portant des fruits sont choisis comme objets et numérotés à la peinture rouge pour éviter le rééchantillonnage des individus. Les arbres échantillonnés étaient distants les uns des autres de $100 \mathrm{~m}$ minimum. Un point GPS a été relevé pour chaque arbre. Au total 22 caractères quantitatifs ont fait l'objet d'étude (Tableau 1) sur 77 arbres. Sur l'arbre, in situ, le diamètre à hauteur de poitrine, la hauteur du 
fût et la hauteur de l'arbre ont été mesurés. Cinq grappes par arbre ont été choisies de façon aléatoire pour la mesure ou le comptage de la longueur du pédoncule, l'épaisseur du pédoncule, le nombre de fruits par pédoncule, le poids de 100 graines par grappe, le poids de graines par grappe, le nombre de graines. Sur ces grappes, cinq fruits ont été choisis pour la mesure ou le comptage de la longueur du pédicelle, l'épaisseur du pédicelle, la longueur du fruit, la largeur du fruit, l'épaisseur du fruit (Figure 2), le poids du fruit, le nombre de graines par fruit, le poids de la pulpe et de graines par fruit. Dans chaque fruit, cinq graines ont été choisies de manière aléatoire pour la mesure de la longueur, la largeur et l'épaisseur des graines (Figure 3).

\section{Analyse des données}

Une matrice de données a été constituée par arbre et par site pour chaque paramètre. Une analyse de corrélation entre les variables prises deux à deux a été réalisée suivi d'un test de comparaison des savanes guinéenne, sub-soudanaise et soudanaise échantillonnées pour chacun des caractères à l'aide d'une analyse de variance multivariée (MANOVA) suivi d'une Analyse de Variance à critère (ANOVA 1). Lorsque deux variables sont corrélées, une seule a été retenue pour une analyse multifactorielle (Analyse Factorielle Discriminante, AFD) sur les données centrées réduites pour rechercher les variables les plus discriminantes parmi celles qui ont donné une différence significative entre les sites. Pour confirmer l'ordination des arbres dans le plan factoriel 1 et 2, une Classification Ascendante Hiérarchique $(\mathrm{CAH})$ sur les données centrées réduites a été réalisée.

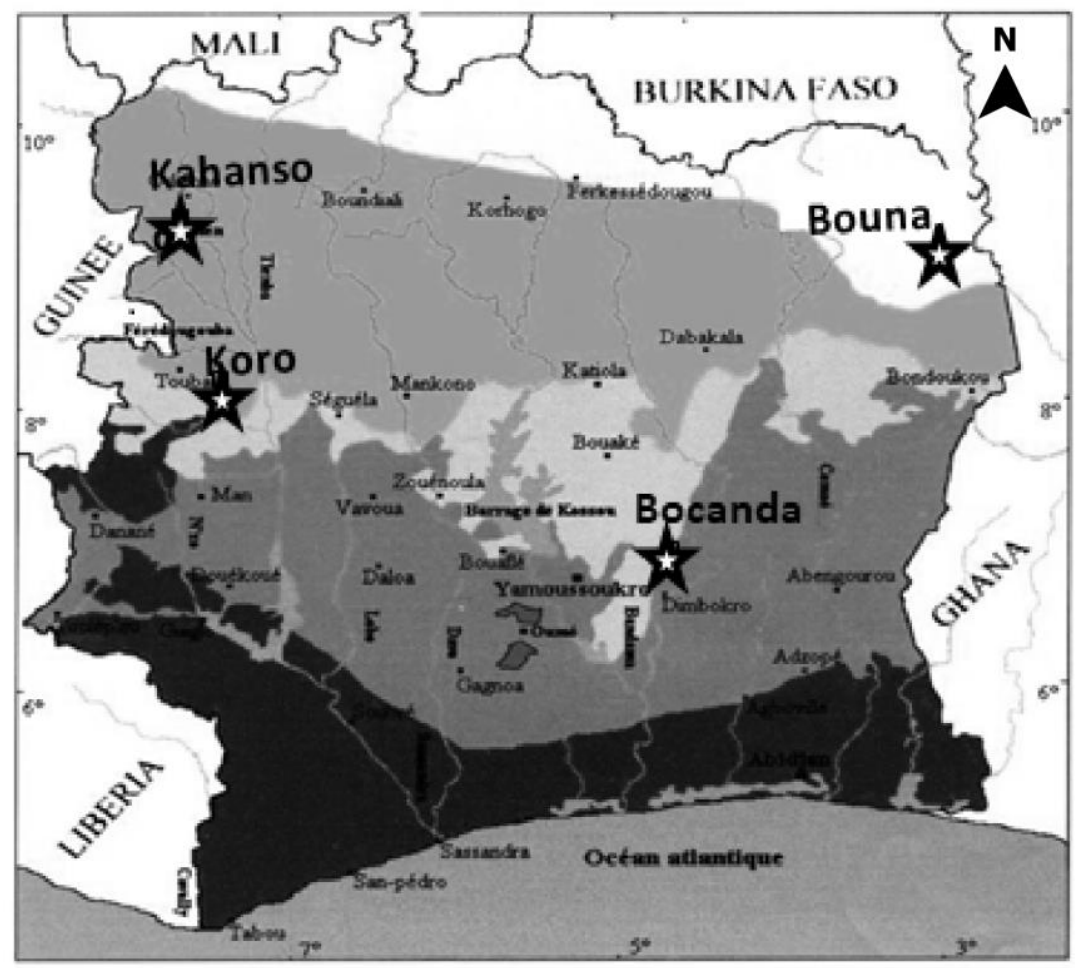

Sites de collecte de Parkia biglobosa

Echelle : 1/400 000

Figure 1: Présentation des sites de collectes de Parkia biglobosa dans les trois types de savane de Cote d'Ivoire.

Du Nord au Sud, les zones de végétation Savane soudanaise, Savane sub-soudanaise, Savane guinéenne, Forêt mésophile, Forêt ombrophile. 
Tableau 1: Codification des paramètres étudiés.

\begin{tabular}{|c|c|}
\hline Paramètres & Libellés \\
\hline Dia & Diamètre du tronc en $\mathrm{cm}$ \\
\hline Hf & Hauteur du fût en $\mathrm{m}$ \\
\hline Ht & Hauteur totale de l'arbre en m \\
\hline NGr & Nombre de graines par fruit \\
\hline LGr & Longueur de la graine en $\mathrm{mm}$ \\
\hline IGr & Largeur de la graine en mm \\
\hline EpGr & Epaisseur de la graine en $\mathrm{mm}$ \\
\hline PGrFr & Poids de graines par fruit en $\mathrm{g}$ \\
\hline P100 & Poids de 100 graines en $\mathrm{g}$ \\
\hline LFr & Longueur du fruit en $\mathrm{cm}$ \\
\hline Lpéd & Longueur du pédicelle en $\mathrm{cm}$ \\
\hline IFr & Largeur du fruit \\
\hline PFr & Poids du fruit en $g$ \\
\hline EpPédic & Epaisseur du pédicelle en mm \\
\hline EpFr & Epaisseur du fruit en mm \\
\hline Ppulpe & Poids de la pulpe en $g$ \\
\hline NFrPedon & Nombre de fruits par pédoncule \\
\hline $\mathbf{L}$ pedon & Longueur du pédoncule en $\mathrm{cm}$ \\
\hline EpPedon & Epaisseur du pédoncule en mm \\
\hline PGrGrap & Poids de graines par grappe \\
\hline P100Grap & Poids de 100 graines par grappe en $\mathrm{g}$ \\
\hline NGrGrap & Nombre de graines par grappe \\
\hline
\end{tabular}




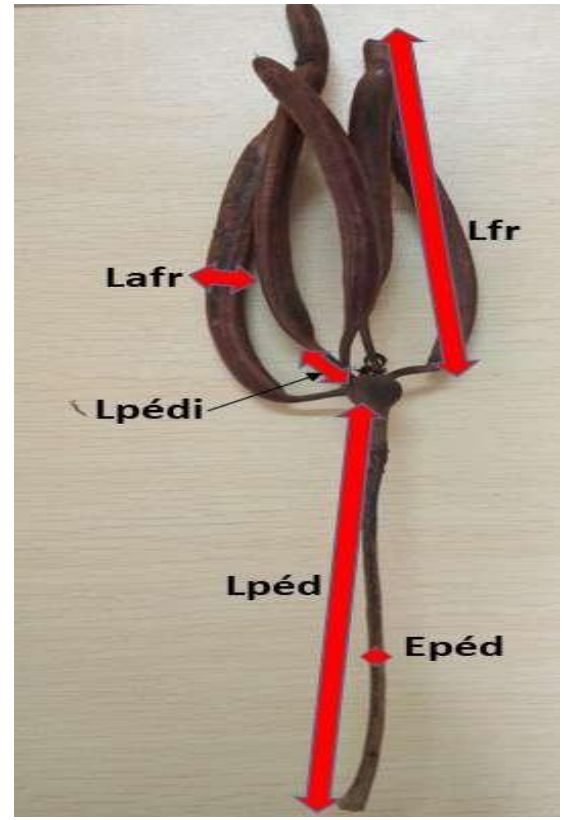

Figure 2 (Matériel supplémentaire) : Illustration des mesures prises sur les grappes de Parkia biglobosa (Jacq.) R. Br. ex G. Don, Fabaceae).

Epéd : épaisseur du pédoncule ; Lafr : largeur du fruit ; Lfr : longueur du fruit ; Lpéd : longueur du pédoncule; Lpédi : longueur du pédicelle.

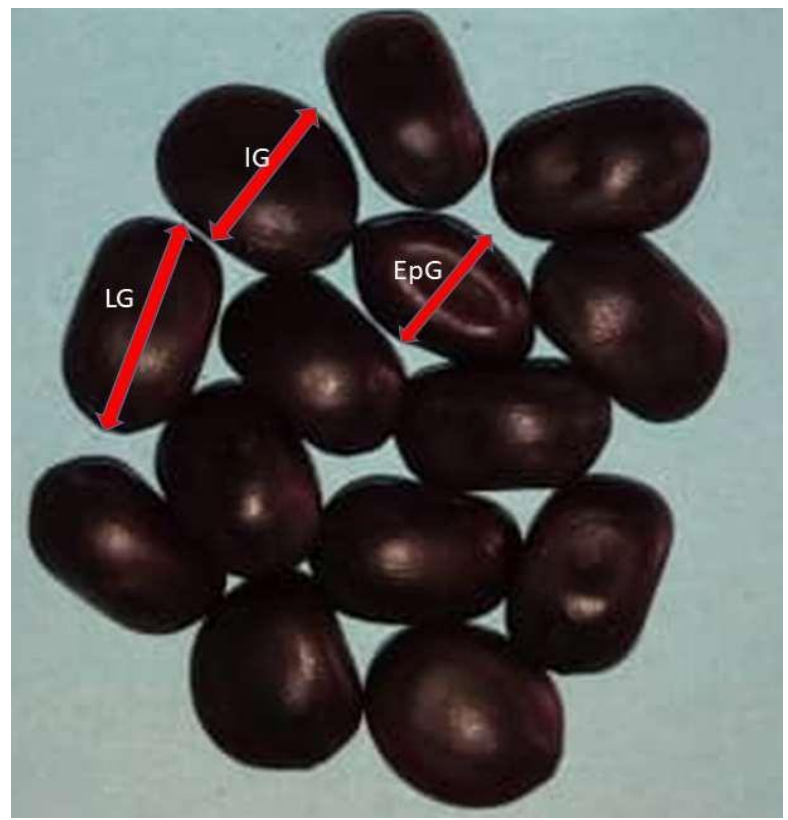

Figure 3 (Matériel supplémentaire): Illustration des mesures prises sur les graines de Parkia biglobosa (Jacq.) R. Br. ex G. Don, Fabaceae).

EpG : épaisseur de la graine ; LG : longeurn de la graine ; IG : largeur de la graine. 


\section{RESULTATS}

La matrice de corrélation (Tableau 2) a révélé une corrélation positive entre quatre paires de variables (poids de graines par fruit et nombre de graines par fruit avec $r=+0,776$, poids de graines par fruit et poids de graines par grappe avec $r=+0,72$, poids de graines par grappe et nombre de graines par grappe avec $\mathrm{r}=+0,79$ et enfin poids de fruit contre poids de pulpe avec $r=+0,74)$. Dans les analyses multifactorielles, les paramètres nombre de graines par fruit, poids de fruit, nombre de graines par grappe et poids de graines par grappe ont été exclus.

Sur les 18 variables restantes, neuf présentant une différence significative ont été utilisés pour les analyses multivariées (AFD et $\mathrm{CAH}$ ) (Figure 4 ; Figure 5 ; Figure 6) après une transformation centrée réduite des données.

\section{Variabilité morphologique entre types de savane}

L'ANOVA a révélé une différence significative entre les savanes pour les paramètres hauteur total, épaisseur de la graine, poids de graine par fruit, longueur du fruit, longueur du pédoncule, épaisseur du pédicelle, poids de pulpe par fruit, longueur et épaisseur du pédoncule (Tableau 3). Seul le paramètre épaisseur du pédoncule permet une distinction totale des savanes avec les valeurs les plus élevées pour la savane guinéenne, suivie de de la savane sub-soudanaise et enfin la savane soudanaise. Pour les huit autres paramètres distinctifs, la savane guinéenne présente les plus grandes valeurs pour la longueur du fruit et la longueur du pédoncule. La savane sub-soudanaise présente les valeurs les plus élevées pour l'épaisseur des graines et le poids de pulpe par fruit. Quant à la savane soudanaise, elle présente les plus grandes valeurs moyennes de longueur du pédoncule.

\section{Structuration de la diversité morphologique}

L'analyse factorielle discriminante a révélé un chevauchement de la zone de répartition des arbres des trois types de savane dans le plan factoriel des deux premiers axes (Figure 4) qui expriment la totalité de la variabilité. Les axes 1 et 2 expriment respectivement $72,02 \%$ et $27,98 \%$ de la variation totale. Sur l'axe 1, les individus à l'extrémité gauche appartiennent à la savane guinéenne alors que les individus à l'extrémité droite appartiennent à la savane subsoudanaise. Sur l'axe 2, les individus à l'extrémité basse appartiennent à la savane sub-soudanaise alors que les individus à l'extrémité haute appartiennent à la savane soudanaise (Figure 4).

L'axe 1 est positivement corrélé à la longueur du pédoncule et négativement corrélé à la longueur du fruit et à l'épaisseur $\mathrm{du}$ pédoncule. L'axe 2 est négativement corrélé au poids de la pulpe, à l'épaisseur de la graine et du pédicelle (Figure 4, Tableau 4).

Les deux axes serviront donc à décrire les groupes identifiés. La classification ascendante hiérarchique a révélé trois groupes d'arbres sur la base de la similarité des caractères discriminants (Figure 6). En fonction de leur origine, le groupe I est constitué d'arbres provenant de Bouna (savane soudanaise) et Koro (savane subsoudanaise), le groupe II qui est de loin le plus important, est formé d'arbres provenant des trois types de savanes et des quatre sites. Le groupe III est constitué d'arbres de Bocanda (savane guinéenne), Koro et Kahanso (savane sub-soudanaise). 


\section{C. KOUONON et al. / Int. J. Biol. Chem. Sci. 14(2): 555-569, 2020}

Tableau 2: Matrice de corrélation: Matériel supplémentaire.

\begin{tabular}{|c|c|c|c|c|c|c|c|c|c|c|c|c|c|c|c|c|c|c|c|}
\hline Variables & $\begin{array}{c}\text { Zone } \\
\text { agroécologique- } \\
\text { SavGui } \\
\end{array}$ & $\begin{array}{c}\text { Zone } \\
\text { agroécologique- } \\
\text { SavSoud } \\
\end{array}$ & $\begin{array}{c}\text { Zone } \\
\text { agroécologique- } \\
\text { SavSub } \\
\end{array}$ & $\begin{array}{l}\text { Dia } \\
(\mathrm{cm})\end{array}$ & $\mathrm{Ht}(\mathrm{m})$ & $\begin{array}{l}\mathrm{LGr} \\
(\mathrm{mm})\end{array}$ & $\begin{array}{l}1 \mathrm{Gr} \\
(\mathrm{mm}) 2\end{array}$ & $\begin{array}{l}\text { EpGr } \\
(\mathrm{mm})\end{array}$ & $\begin{array}{l}\mathrm{PGrFr} \\
(\mathrm{g})\end{array}$ & P100 & $\begin{array}{l}\mathrm{LFr} \\
(\mathrm{Cm})\end{array}$ & $\begin{array}{l}\text { Lpéd } \\
(\mathrm{cm})\end{array}$ & $\begin{array}{l}\text { milLarfr } \\
(\mathrm{mm})\end{array}$ & $\begin{array}{c}\mathrm{PFr} \\
(\mathrm{g})\end{array}$ & $\begin{array}{l}\text { EpPédic } \\
\text { (mm) }\end{array}$ & $\begin{array}{l}\text { EpFr } \\
(\mathrm{mm})\end{array}$ & NFrPedon & $\begin{array}{l}\text { L pedon } \\
(\mathrm{cm})\end{array}$ & $\begin{array}{l}\text { EpPedon } \\
(\mathrm{mm})\end{array}$ \\
\hline $\begin{array}{l}\text { Zone } \\
\text { agroécologique- } \\
\text { SavGui }\end{array}$ & 1,0000 & $-0,3900$ & $-0,6133$ & $-0,0186$ & $-0,3143$ & $-0,0962$ & 0,0374 & $-0,3019$ & 0,0386 & 0,0199 & 0,3761 & 0,5223 & $-0,5697$ & 0,0159 & $-0,0236$ & $-0,2627$ & 0,2221 & $-0,3600$ & 0,4385 \\
\hline $\begin{array}{l}\text { Zone } \\
\text { agroécologique- } \\
\text { SavSoud }\end{array}$ & $-0,3900$ & 1,0000 & $-0,4880$ & 0,1422 & $-0,0673$ & $-0,1168$ & $-0,1355$ & $-0,1125$ & $-0,2852$ & $-0,0474$ & $-0,2486$ & $-0,0097$ & 0,5453 & $-0,3294$ & $-0,4225$ & 0,0787 & $-0,0486$ & 0,4639 & $-0,4124$ \\
\hline $\begin{array}{l}\text { Zone } \\
\text { agroécologique- } \\
\text { SavSub }\end{array}$ & $-0,6133$ & $-0,4880$ & 1,0000 & $-0,1043$ & 0,3557 & 0,1914 & 0,0808 & 0,3827 & 0,2081 & 0,0218 & $-0,1432$ & $-0,4868$ & 0,0723 & 0,2675 & 0,3848 & 0,1814 & $-0,1688$ & $-0,0567$ & $-0,0620$ \\
\hline $\operatorname{Dia}(\mathrm{cm})$ & $-0,0186$ & 0,1422 & $-0,1043$ & 1,0000 & 0,4296 & 0,2382 & 0,1999 & 0,1196 & 0,2866 & 0,2396 & 0,2077 & 0,0451 & 0,1087 & 0,1805 & 0,2740 & $-0,1924$ & $-0,2873$ & 0,0963 & $-0,1531$ \\
\hline $\mathrm{Ht}(\mathrm{m})$ & $-0,3143$ & $-0,0673$ & 0,3557 & 0,4296 & 1,0000 & 0,0563 & 0,0540 & 0,3552 & 0,2280 & 0,1450 & 0,1463 & $-0,2203$ & 0,2108 & 0,3003 & 0,2603 & 0,2126 & 0,0168 & 0,0649 & 0,0679 \\
\hline $\mathrm{LGr}(\mathrm{mm})$ & $-0,0962$ & $-0,1168$ & 0,1914 & 0,2382 & 0,0563 & 1,0000 & 0,6873 & 0,2783 & 0,6894 & 0,6592 & 0,2305 & 0,0208 & 0,1505 & 0,5429 & 0,4251 & 0,0407 & $-0,1863$ & $-0,1784$ & 0,0415 \\
\hline $1 \mathrm{Gr}(\mathrm{mm})$ & 0,0374 & $-0,1355$ & 0,0808 & 0,1999 & 0,0540 & 0,6873 & 1,0000 & 0,1479 & 0,5258 & 0,6282 & 0,5086 & 0,1819 & $-0,1196$ & 0,5565 & 0,3799 & $-0,0153$ & $-0,1787$ & $-0,0911$ & $-0,0321$ \\
\hline $\mathrm{EpGr}(\mathrm{mm})$ & $-0,3019$ & $-0,1125$ & 0,3827 & 0,1196 & 0,3552 & 0,2783 & 0,1479 & 1,0000 & 0,4874 & 0,2466 & $-0,0048$ & $-0,1264$ & 0,0852 & 0,3074 & 0,5638 & 0,4417 & 0,1263 & $-0,1579$ & 0,3768 \\
\hline $\operatorname{PGrFr}(\mathrm{g})$ & 0,0386 & $-0,2852$ & 0,2081 & 0,2866 & 0,2280 & 0,6894 & 0,5258 & 0,4874 & 1,0000 & 0,6285 & 0,5319 & 0,0313 & $-0,0012$ & 0,6874 & 0,6107 & 0,0666 & $-0,0400$ & $-0,3902$ & 0,3115 \\
\hline P100 & 0,0199 & $-0,0474$ & 0,0218 & 0,2396 & 0,1450 & 0,6592 & 0,6282 & 0,2466 & 0,6285 & 1,0000 & 0,3313 & $-0,0779$ & 0,0583 & 0,5930 & 0,3895 & 0,2100 & $-0,1781$ & $-0,1530$ & 0,0342 \\
\hline $\operatorname{LFr}(\mathrm{Cm})$ & 0,3761 & $-0,2486$ & $-0,1432$ & 0,2077 & 0,1463 & 0,2305 & 0,5086 & $-0,0048$ & 0,5319 & 0,3313 & 1,0000 & 0,3495 & $-0,2740$ & 0,6291 & 0,2900 & $-0,0665$ & 0,0302 & $-0,1905$ & 0,2109 \\
\hline Lpéd $(\mathrm{cm})$ & 0,5223 & $-0,0097$ & $-0,4868$ & 0,0451 & $-0,2203$ & 0,0208 & 0,1819 & $-0,1264$ & 0,0313 & $-0,0779$ & 0,3495 & 1,0000 & $-0,2731$ & $-0,0005$ & $-0,1425$ & $-0,1783$ & 0,1392 & $-0,0277$ & 0,2579 \\
\hline millarff (mm) & $-0,5697$ & 0,5453 & 0,0723 & 0,1087 & 0,2108 & 0,1505 & $-0,1196$ & 0,0852 & $-0,0012$ & 0,0583 & $-0,2740$ & $-0,2731$ & 1,0000 & 0,0204 & $-0,1141$ & 0,2575 & $-0,1172$ & 0,3424 & $-0,2805$ \\
\hline $\operatorname{PFr}(\mathrm{g})$ & 0,0159 & $-0,3294$ & 0,2675 & 0,1805 & 0,3003 & 0,5429 & 0,5565 & 0,3074 & 0,6874 & 0,5930 & 0,6291 & $-0,0005$ & 0,0204 & 1,0000 & 0,5946 & 0,3694 & $-0,1849$ & $-0,1039$ & 0,1326 \\
\hline EpPédic (mm) & $-0,0236$ & $-0,4225$ & 0,3848 & 0,2740 & 0,2603 & 0,4251 & 0,3799 & 0,5638 & 0,6107 & 0,3895 & 0,2900 & $-0,1425$ & $-0,1141$ & 0,5946 & 1,0000 & 0,2533 & $-0,1946$ & $-0,2018$ & 0,3539 \\
\hline $\mathrm{EpFr}(\mathrm{mm})$ & $-0,2627$ & 0,0787 & 0,1814 & $-0,1924$ & 0,2126 & 0,0407 & $-0,0153$ & 0,4417 & 0,0666 & 0,2100 & $-0,0665$ & $-0,1783$ & 0,2575 & 0,3694 & 0,2533 & 1,0000 & 0,2104 & 0,0534 & 0,2713 \\
\hline NFrPedon & 0,2221 & $-0,0486$ & $-0,1688$ & $-0,2873$ & 0,0168 & $-0,1863$ & $-0,1787$ & 0,1263 & $-0,0400$ & $-0,1781$ & 0,0302 & 0,1392 & $-0,1172$ & $-0,1849$ & $-0,1946$ & 0,2104 & 1,0000 & $-0,2216$ & 0,5922 \\
\hline L pedon $(\mathrm{cm})$ & $-0,3600$ & 0,4639 & $-0,0567$ & 0,0963 & 0,0649 & $-0,1784$ & $-0,0911$ & $-0,1579$ & $-0,3902$ & $-0,1530$ & $-0,1905$ & $-0,0277$ & 0,3424 & $-0,1039$ & $-0,2018$ & 0,0534 & $-0,2216$ & 1,0000 & $-0,5139$ \\
\hline EpPedon (mm) & 0,4385 & $-0,4124$ & $-0,0620$ & $-0,1531$ & 0,0679 & 0,0415 & $-0,0321$ & 0,3768 & 0,3115 & 0,0342 & 0,2109 & 0,2579 & $-0,2805$ & 0,1326 & 0,3539 & 0,2713 & 0,3922 & $-0,5139$ & 1,0000 \\
\hline
\end{tabular}




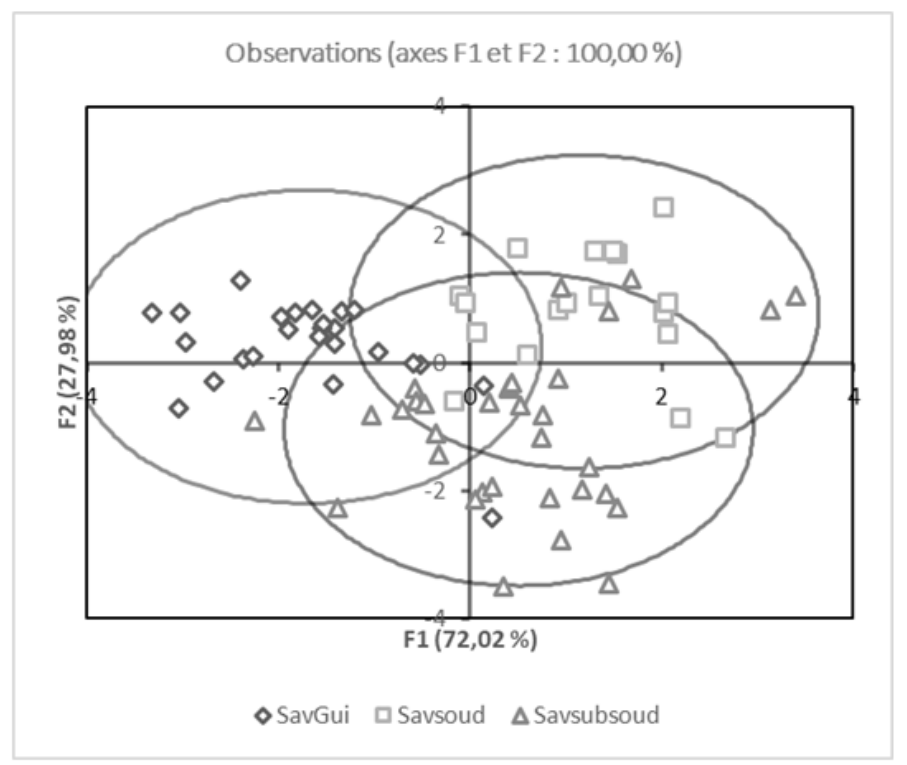

Figure 4: Ordination des arbres dans le plan factoriel des axes 1 et 2 d'une analyse factorielle discriminante.

SavGui : savane guinéenne ; Savsoud : Savane soudanaise ; Savsubsoud : Savane sub-soudanaise.

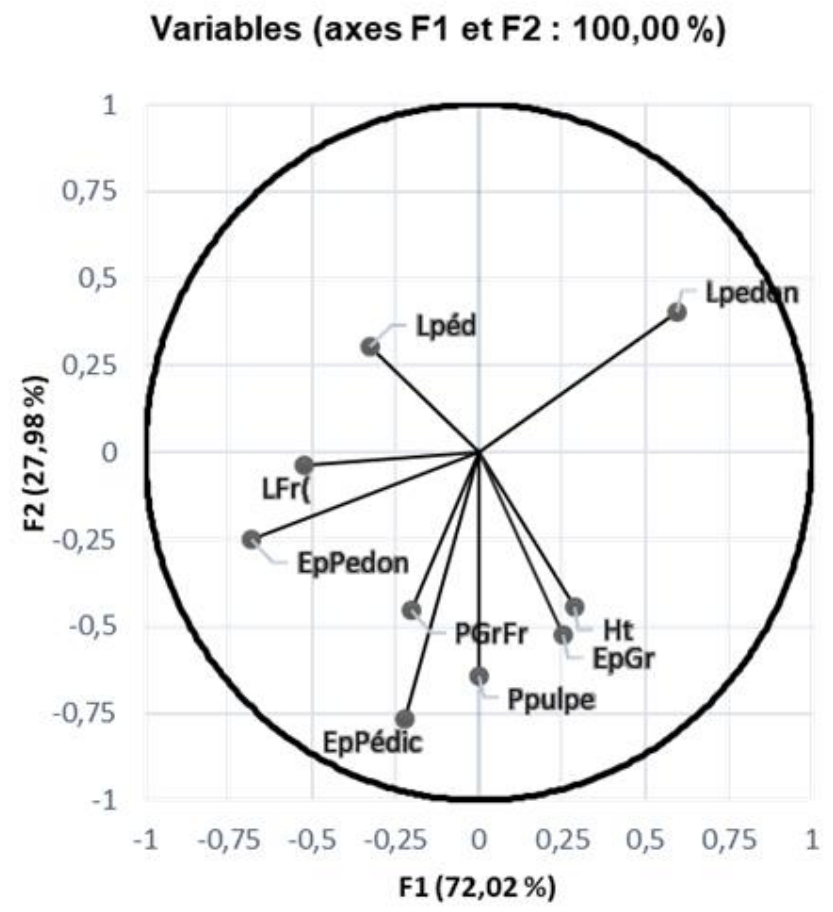

Figure 5 : Cercle des corrélations des variables discriminantes dans le plan factoriel des axes 1 et 2. Ht : Hauteur arbre ; Lpéd : longueur pédicelle ; Lpedon : longueur pédoncule ; LFr : longueur fruit ; EpPedon : épaisseur pédoncule ; PGrFr : poids de graine par fruit ; EpGr : épaisseur graine; Ppulpe : poids pulpe ; EpPédic : épaisseur pédicelle. 


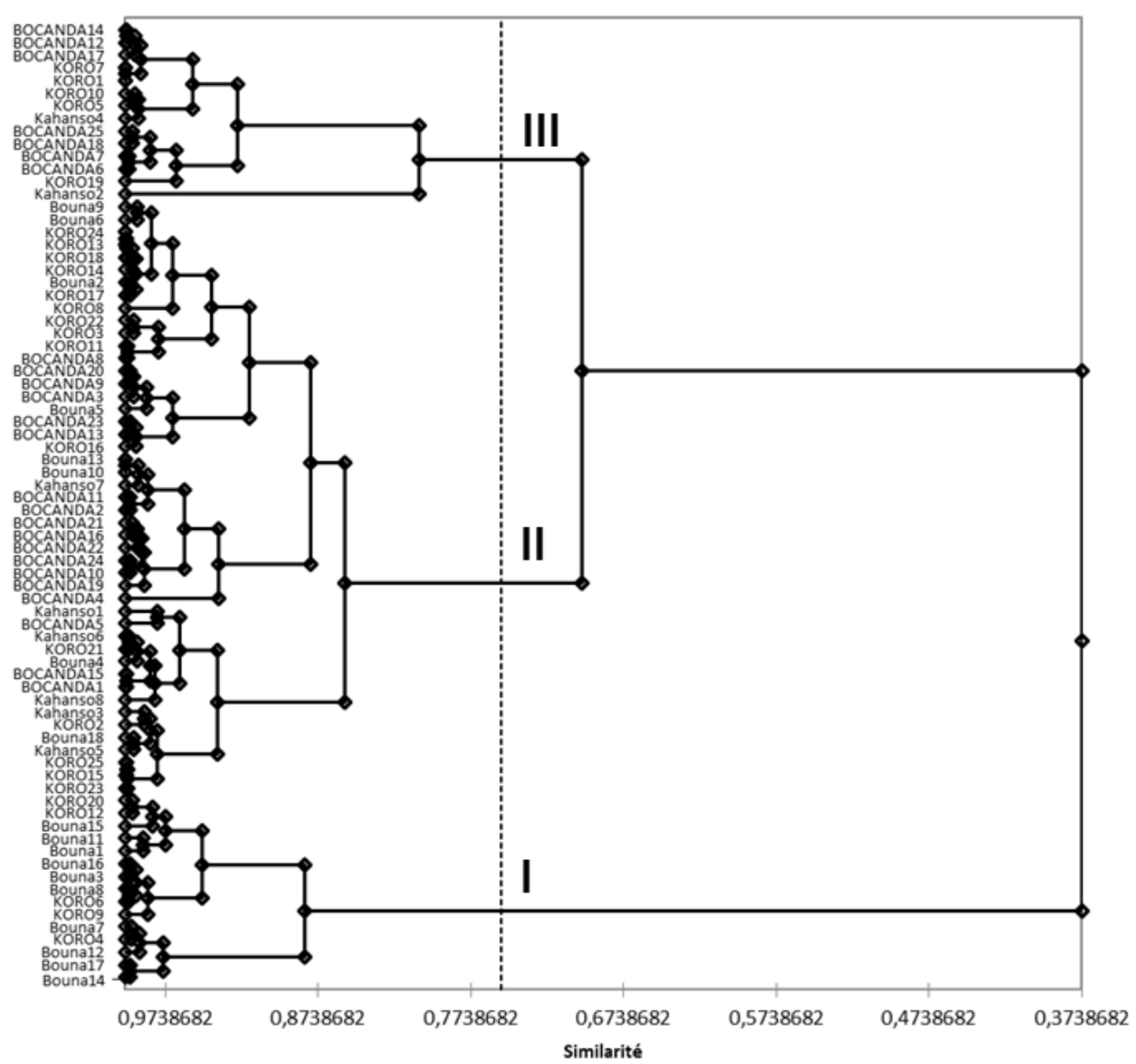

Figure 6: Dendrogramme de la classification ascendante hiérarchique des 77 individus sur la base des caractères discriminants en trois groupes (I, II et III).

Tableau 3: Récapitulatif des moyennes et écart-types par type de savane et test de comparaison des savanes.

\begin{tabular}{|c|c|c|c|c|c|}
\hline Paramètres & $\mathbf{F}$ & $\operatorname{Pr}>F$ & Subsoudanaise & Guinéenne & Soudanaise \\
\hline Dia $(\mathrm{cm})$ & 0,8299 & 0,4402 & $41,87 \pm 17,58$ & $42,45 \pm 17,58$ & $48,38 \pm 19,7$ \\
\hline $\mathrm{Hf}(\mathrm{m})$ & 2,6464 & 0,0777 & $3,62 \pm 2,16$ & $2,6 \pm 1,48$ & $2,85 \pm 1,2$ \\
\hline $\mathbf{H t}(\mathbf{m})$ & 6,0075 & 0,0038 & $10,68 \pm 2,46^{a}$ & $8,5 \pm 2,27^{b}$ & $9,34 \pm 2,5^{\mathrm{ab}}$ \\
\hline NGr & 1,8780 & 0,1602 & $15,62 \pm 2,43$ & $15,91 \pm 1,93$ & $14,51 \pm 3,02$ \\
\hline LGr (mm) & 1,4169 & 0,2491 & $9,2 \pm 0,92$ & $8,85 \pm 1,11$ & $8,78 \pm 0,87$ \\
\hline $1 \mathrm{Gr}(\mathrm{mm})$ & 0,6936 & 0,5030 & $7,53 \pm 0,88$ & $7,5 \pm 0,76$ & $7,26 \pm 0,85$ \\
\hline EpGr (mm) & 6,6274 & 0,0023 & $5,05 \pm 0,77^{a}$ & $4, \mathbf{4 5} \pm 0,52^{b}$ & $4,61 \pm 0,58^{b}$ \\
\hline PGrFr (g) & $\mathbf{3 , 5 0 3 7}$ & $\mathbf{0 , 0 3 5 2}$ & $3,24 \pm 0,91^{\mathrm{a}}$ & $3,08 \pm 0,82^{a b}$ & $2,59 \pm 0,73^{b}$ \\
\hline P100 & 0,0821 & 0,9213 & $21,16 \pm 3,81$ & $21,18 \pm 3,65$ & $20,7 \pm 5,8$ \\
\hline $\operatorname{LFr}(\mathrm{Cm})$ & 6,6282 & 0,0023 & $21,88 \pm 3,33^{b}$ & $24,45 \pm 3,58^{\mathrm{a}}$ & $20,84 \pm 3,46^{b}$ \\
\hline \multirow[t]{2}{*}{ Lpéd (cm) } & 4,1847 & 0,0190 & $3,29 \pm 1,33^{b}$ & $4,17 \pm 1,08^{\mathrm{a}}$ & $3,63 \pm 0,91^{\mathrm{ab}}$ \\
\hline & & & 564 & & \\
\hline
\end{tabular}




\begin{tabular}{|c|c|c|c|c|c|}
\hline lFr(mm) & 2,5668 & 0,0837 & $19,27 \pm 2,22$ & $18,77 \pm 1,97$ & $17,91 \pm 1,8$ \\
\hline $\operatorname{PFr}(\mathrm{g})$ & 5,1414 & 0,0081 & $14,73 \pm 3,97$ & $13,7 \pm 3,13$ & $11,47 \pm 2,91$ \\
\hline EpPédic(mm) & 10,3169 & 0,0001 & $4,17 \pm 0,7^{\mathrm{a}}$ & $3,87 \pm 0,48^{a}$ & $3,4 \pm 0,45^{b}$ \\
\hline $\operatorname{EpFr}(\mathrm{mm})$ & 0,8140 & 0,4471 & $10,32 \pm 1,78$ & $9,85 \pm 1,11$ & $10,23 \pm 0,96$ \\
\hline Ppulpe(g) & 6,4297 & 0,0027 & $3,19 \pm 1,1^{\mathrm{a}}$ & $2,53 \pm 0,97^{b}$ & $2,25 \pm 0,68^{b}$ \\
\hline NFrPedon & 1,4558 & 0,2399 & $9,1 \pm 4,71$ & $10,93 \pm 3,5$ & $9,29 \pm 4,33$ \\
\hline $\mathrm{L} \operatorname{pedon}(\mathrm{cm})$ & 11,0382 & $<0,0001$ & $15,86 \pm 6^{b}$ & $13,4 \pm 3,7^{b}$ & $20,83 \pm 5,17^{\mathrm{a}}$ \\
\hline $\operatorname{EpPedon}(\mathbf{m m})$ & 12,8915 & $<0,0001$ & $5,78 \pm 0,94^{b}$ & $6,44 \pm 0,73^{a}$ & $5,16 \pm 0,71^{\mathrm{c}}$ \\
\hline PGrGrap(g) & 1,9453 & 0,1503 & $11,17 \pm 3,38$ & $11,16 \pm 3,4$ & $9,25 \pm 4,2$ \\
\hline P100Grap(g) & 1,6647 & 0,1964 & $26,65 \pm 19,45$ & $22,35 \pm 3,1$ & $20,05 \pm 3,73$ \\
\hline NGrGrap & 1,3737 & 0,2596 & $51,06 \pm 17,66$ & $49,93 \pm 12,97$ & $43,72 \pm 14,54$ \\
\hline
\end{tabular}

Les lignes en gras présentent les tests significatifs au seuil 0,05. Les exposants a, b et c indiquent les moyennes qui sont statistiquement différentes. Deux moyennes portant des lettres différentes sont différentes dans l'ordre a, le plus grand puis b puis c. Dia, Diamètre du tronc en cm; Hf, Hauteur du fût en m; Ht, Hauteur totale de l'arbre en m ; NGr, Nombre de graines par fruit ; LGr, Longueur de la graine en mm; IGr, Largeur de la graine en mm; EpGr, Epaisseur de la graine en mm ; PGrFr, Poids de graines par fruit en g ; P100 ; Poids de 100 graines en g : LFr, Longueur du fruit en cm ; Lpéd, Longueur du pédicelle en $\mathrm{cm}$; IFr, Largeur du fruit ; PFr, Poids du fruit en g ; EpPédic, Epaisseur du pédicelle en mm ; EpFr, Epaisseur du fruit en mm ; Ppulpe, Poids de la pulpe en g ; NFrPedon, Nombre de fruits par pédoncule ; L pedon, Longueur du pédoncule en $\mathrm{cm}$; EpPedon, Epaisseur du pédoncule en mm; PGrGrap ; Poids de graines par grappe ; P100Grap, Poids de 100 graines par grappe en g ; NGrGrap, Nombre de graines par grappe

Tableau 4: Valeurs propres, pourcentage de discrimination des axes 1 et 2, suivis des coefficients de corrélations entre variables et axes.

\begin{tabular}{lrr}
\hline & F1 & F2 \\
\hline Valeur propre & 1,59 & 0,62 \\
Discrimination (\%) & 72,02 & 27,98 \\
\hline $\mathrm{Ht}$ & 0,29 & $-0,45$ \\
EpGr & 0,26 & $\mathbf{- 0 , 5 3}$ \\
PGrFr & $-0,20$ & $-0,46$ \\
LFr & $\mathbf{- 0 , 5 2}$ & $-0,04$ \\
Lpéd & $-0,32$ & 0,31 \\
EpPédic & $-0,22$ & $\mathbf{- 0 , 7 7 ^ { * }}$ \\
Ppulpe & 0,00 & $\mathbf{- 0 , 6 4}$ \\
Lpedon & $\mathbf{0 , 6 0}$ & 0,40 \\
EpPedon & $\mathbf{- 0 , 6 8}$ & $-0,25$ \\
\hline
\end{tabular}

Les valeurs précédées du signe * indiquent une corrélation significative.

Ht : Hauteur totale de l'arbre en m; EpGr : Epaisseur de la graine en mm; PGrFr : Poids de graines par fruit en g; LFr : Longueur du fruit en $\mathrm{cm}$; Lpéd : Longueur du pédicelle en $\mathrm{cm}$; EpPédic : Epaisseur du pédicelle en mm ; Ppulpe : Poids de la pulpe en g; Lpedon : Longueur du pédoncule en cm ; EpPedon : Epaisseur du pédoncule en mm. 


\section{DISCUSSION}

Sur les 22 paramètres étudiés, une corrélation a été détectée entre la variable poids de graines par fruit et les variables nombre de graines par fruit et poids de graines par grappe, entre les variables poids de graines par grappe et nombre de graines par grappe et entre les variables poids de fruit et poids de pulpe par fruit. Certes les caractères morphologiques sont les premiers marqueurs pour estimer la variabilité génétique mais du fait de la corrélation qui peut exister entre ces caractères, il faut prendre soin d'éviter de considérer des paramètres corrélés. Ce qui pourrait être une source de biais dans l'estimation de la structure géographique de la variabilité surtout dans les analyses multivariées. Ainsi, la précaution a été prise de ne considérer que des paramètres non corrélés entre eux pour l'analyse multivariée.

\section{Descripteurs morphologiques et évaluation de la variabilité phénotypique}

Dix-huit paramètres sur les 22 étudiés, ont été soumis au test de comparaison des trois types de savane. La moitié de ces paramètres a montré une différence significative entre les savanes. Trois paramètres (épaisseur de pédicelle, longueur de pédoncule et épaisseur de pédoncule) montrent une différence très hautement significative $(\mathrm{p}<0,001)$ entre les savanes mais seule l'épaisseur du pédoncule révèle une distinction totale. Quatre paramètres discriminants (hauteur totale de l'arbre, épaisseur des graines, longueur des fruits, et poids de pulpe) montrent une différence hautement significative $(\mathrm{p}<0,01)$ entre savanes et deux paramètres (poids de graines par fruit et longueur du pédicelle) révèlent une différence significative $(\mathrm{p}<0,05)$ entre savanes. Majoritairement, les organes des fruits et graines (Millogo, 2014) se révèlent comme de bons descripteurs de P. biglobosa et pourraient servir pour une évaluation en routine de la variabilité morphologique de l'espèce. Ces paramètres quantitatifs seraient des marqueurs intéressants pour la sélection variétale (Teklehaimanot, 2004), même si l'héritabilité de ses marqueurs n'est pas encore définie. Les paramètres discriminants révélés par cette étude peuvent servir à catégoriser les arbres de P. biglobosa de Côte d'Ivoire dans un but de sélection variétale pour répondre au mieux aux divers besoins des populations.

\section{Provenance et structure de la variabilité morphologique}

Les trois types de savanes se caractérisent par des régimes de pluies et de températures différents, ce qui a pour conséquence des végétations contrastées. Les facteurs climatiques pourraient exercer une différenciation des paramètres phénotypiques.

La CAH a regroupé les individus en trois types d'arbres. Le groupe I est formé d'arbres de la savane sub-soudanaise et soudanaise. Le groupe III est constitué d'arbres de savanes guinéenne et subsoudannaise alors que le groupe II est formé d'arbres des trois types de savane. La distribution géographique des différents groupes n'est pas bien tranchée comme l'ont observée Amusa et al. (2014) avec des marqueurs RADP. Il y a des zones de transition entre les différents types. Tout se passe comme si une diversité caractéristique des savanes du Sud (guinéenne), plus humides et de celles du Nord (soudanaise), plus sèche se différencie avec une zone de transition (savane sub-soudanaise). Ces résultats sont similaires à ceux obtenus par Millogo (2014). En effet, chez P. biglobosa, il y aurait une variation phénotypique, selon les origines, due à l'environnement et à la différence génétique suivant la latitude quand bien même cette variation est moins importante (Sina, 2006, Adesoye et al., 2013, Amusa et al., 2014) que celle qui subsiste entre individus d'une même population. Des résultats similaires de structure géographique de variation phénotypique ont été obtenus sur des Fabaceae en Côte d'Ivoire sur Pterocarpus erinaceus Poir. (Goba et al., 2019) et au Mali sur Detarium microcarpum Guill. \& Perr. (Kouyaté, 2005). 


\section{Caractéristiques phénotypiques et types de savane}

La similarité entre arbres a révélé trois groupes d'individus dans les savanes de Côte d'Ivoire. P. biglobosa est structuré selon un gradient Sud-Nord (Lompo et al., 2017) et un groupe de transition. En effet, les individus classés dans le groupe III de la CAH appartiennent à la savane guinéenne (plus au Sud) et sub-soudanaise et ceux du groupe I appartiennent à la savane soudanaise (plus au Nord) et sub-soudanaise. La zone subsoudanaise est un mélange des phénotypes du Nord et ceux du Sud. Il y a donc une variation phénotypique géographique des arbres du néré comme l'ont montré Koura et al. $\left(2013^{\mathrm{b}}\right)$ à travers la variabilité phénotypique au Bénin. Les arbres provenant de la savane guinéenne peuvent être décrits comme ayant de longs fruits, longs et épais pédoncules, épais pédicelles. Les paramètres à faibles modalités en savane guinéenne sont: poids de pulpe, épaisseur de graine, hauteur d'arbre et longueur de pédoncule. Les arbres de la savane sub-soudanaise ont des hauteurs, épaisseur de graines et pédicelles, poids de graines et de pulpe par fruit élevés en moyennes. Pour les paramètres épaisseur du pédoncule, longueur de fruit, de pédicelle et de pédoncule, les moyennes sont plus faibles. Les arbres en savane soudanaise sont caractérisés par un long pédoncule, des arbres hauts alors que les paramètres épaisseur de pédoncule, pédicelle et graine, longueur de fruit, poids de graine et pulpe par fruit sont faibles. Sur la base des groupes d'arbres révélés et des caractères discriminants déterminés, des sélections variétales peuvent être initiées pour l'amélioration de différents caractères afin de répondre au mieux aux usages des populations.

\section{Conclusion}

Les paramètres morphologiques ont permis de structurer les arbres des trois types de savanes en deux groupes principaux et un groupe de transition composé d'arbres des trois origines. Les paramètres les plus discriminants pour évaluer la variabilité des arbres de P. biglobosa sont: la hauteur de l'arbre, l'épaisseur de la graine, du pédicelle, du pédoncule, la longueur du pédicelle, du pédoncule, du fruit, le poids de graine et de pulpe par fruit. Ces paramètres peuvent servir à la sélection variétale et à l'estimation des paramètres phénotypes pour une préservation de la ressource P. biglobosa en Côte d'Ivoire. Les arbres qui ont les caractéristiques les plus contrastés devraient servir de point de départ pour la sélection variétale.

\section{CONFLIT D'INTERETS}

Les auteurs déclarent ne connaitre aucun conflit d'intérêt relatif à l'acquisition et à l'analyse des données ainsi qu'à la rédaction du présent article.

\section{CONTRIBUTIONS DES AUTEURS}

LCK: Conception, collecte des données, analyses des données, rédaction de l'article. KGK: Conception, collecte des données, analyses des données, rédaction de l'article. KA: Révision de l'article. ADSM: Collecte des données. KAEG: Collecte et analyses des données. Tous les auteurs ont lu et approuvé la version finale de ce travail.

\section{REMERCIEMENTS}

Les auteurs tiennent à remercier
toutes les personnes (communautés
villageoises et guides) rencontrées lors de cette
étude et qui en ont facilité l'issue.

\section{RÉFÉRENCES}

Adesoye I, Ogunremi CO, Aina OO. 2013. Genetic variation and heritability of seedling traits in African locust Bean Parkia biglobosa (Jacq.) R.Br. Ex G. Don. Legume Research, 36(2): 89-97.

Amusa O, Adesoye A, Ogunkanmi A, Omoche O, Olowe O, Akinyosoye S, Omodele T. 2014. Genetic diversity of Parkia biglobosa from different agroecological zones of Nigeria using RAPD Markers. International Journal of Biodiversity, 2014: 1-6. DOI: https://doi.org/10.1155/2014/457309.

Avana-Tientcheu MLA, Keouna S, Dongock Nguemo D, Mouga Masdewel B. 2019. 
Structure des peuplements et potentiel de domestication de Parkia biglobosa dans la région de Tandjilé-Ouest (Tchad). International Journal of Biological and Chemical Sciences, 13(1): 219-236. DOI:

https://dx.doi.org/10.4314/ijbcs.v13i1.19

Babalola FD. 2012. Evaluation of the Marketing Chain of Parkia biglobosa (Jacq. Benth) R. Br. ex G. Don in SouthWest Nigeria. International Journal of Basic and Applied Sciences, 1(3): 210217.

Bayala J, Mando A, Teklehaimanot Z, Ouedraogo SJ. 2005. Nutrient release from decomposing leaf mulches of karite (Vitellaria paradoxa) and nere (Parkia biglobosa) under semi-arid conditions in Burkina Faso, West Africa. International Information System for the Agricultural Science and Technology, 37(3): 533-539. DOI:

https://doi.org/10.1016/j.soilbio.2004.08. 015.

Camara F, Soro S, Traore S, Brou K, Djè KM. 2016. Caractéristiques biochimiques et microbiologiques de moutardes africaines produites à base de graines fermentées de Parkia biglobosa et de Glycine max, vendues en Côte d'Ivoire. International Journal of Biological and Chemical Sciences, 10(2): 506-518. DOI:

http://dx.doi.org/10.4314/ijbcs.v10i2.5.

Dedehou VFGN, Olounladé PA, Adenilé DA. Alowanou GG, Azando EVB, Hounzangbé-Adoté MS. 2016. A review on medicinal plants of Parkia Biglobosa (Mimosaceae -Fabaceae) and Pterocarpus erinaceus (Leguminosae Papilionoidea). Journal of Medicinal Plants Studies, 4(6): 132-137.

Diarassouba N, Koffi EK, N'Guessan AN, Van Damme P, Sangaré A. 2008. Connaissances locales et leur utilisation dans la gestion des parcs à karité en Côte d'Ivoire. Afrika focus, 21(1) : 77-96.

Goba KAE, Kouonon LC, Koffi KG, Bony BS, Diomandé I, Sié RS. 2019. Morphological Diversity within
Pterocarpus erinaceus Poir. (Fabaceae), an overexploited species in the savannahs of Côte d'Ivoire. American Journal of Plant Sciences, 10(9): 16751688.

DOI: https://doi.org/10.4236/ajps.2019.109119

Guillaumet J-L, Adjanohoun E. 1971. La végétation de la Côte d'Ivoire. In Le Milieu Naturel De La Côte d'Ivoire. ORSTOM : Paris ; 161-263.

Ikabanga DU, Stévart T, Koffi KG, Monthé KFS, Nzigo-Doubindou EC, Dauby G, Souza A, M'Batchi B, Hardy OJ. 2017. Combining morphology and population genetic analysis uncover species delimitation in the widespread african tree genus Santiria. Phytotaxa, 321(2): 166-180.

DOI : http://dx.doi.org/10.11646/phytotaxa.321 .2.2.

Hahn-Hadjali K, Thiombiano A. 2000. Perception des espèces en voie de disparition en milieu Gourmantché (Est $\mathrm{du}$ Burkina Faso). Berichte des Sonderforschungsbereichs, $\quad \mathbf{2 6 8}(14)$ : 285-297.

Koura K, Dissou EF, Ganglo JC. 2013 ${ }^{\text {. }}$. Caractérisation écologique et structurale des parcs à néré [Parkia biglobosa (Jacq.) R. Br. Ex G. Don] du département de la Donga au Nord-Ouest $\mathrm{du}$ Bénin. International Journal of Biological and Chemical Sciences, 7(2): 726-738.

DOI: http://dx.doi.org/10.4314/ijbcs.v7i2.27

Koura K, Mbaide Y, Ganglo JC. 2013 ${ }^{\text {. }}$. Caractéristiques phénotypique et structurale de la population de Parkia biglobosa (Jacq.) R. Br. du Nord-Bénin. International Journal of Biological and Chemical Sciences, 7(6): 2409-2425. DOI: http://dx.doi.org/10.4314/ijbcs.v7i6.19.

Kouyaté AM. 2005. Aspects ethnobotaniques et étude de la variabilité morphologique, biochimique et phénologique de Detarium microcarpum Guill. \& Perr. au Mali. PhD thesis, Ghent University, Belgium, $207 \mathrm{p}$. 
Kouyaté AM, Decalluwé E, Guindo F, Diawara H, Diarra I, N'Diaye I, van Damme P. 2011. Variabilité morphologique du baobab (Adansonia digitata L.) au Mali. Fruits, 66: 247-255.

Kremer A. 2000. Global change and genetic diversity. Revue forestière française, $\mathbf{5 2}$ : 91-98.

Lamien N, Ekué M, Ouedraogo M, Loo J. 2011. Parkia biglobosa, néré : Conservation et utilisation durable des ressources génétiques des espèces ligneuses alimentaires prioritaires de l'Afrique subsaharienne. Bioversity International, Rome, Italie, 8p.

Lassen KM, Kjær ED, Ouédraogo M, Nielsen LR. 2014. Microsatellite primers for Parkia biglobosa (Fabaceae: Mimosoideae) reveal that a single plant sires allseeds per pod. Appl. Pl. Sci., 2: 1400024.

DOI: https://doi.org/10.3732/apps.1400024.

Lompo D, Vinceti B, Gaisberger H, Konrad H, Duminil J, Ouedraogo M, Sina S, Geburek T. 2017. Genetic conservation in Parkia biglobosa (Fabaceae: Mimosoideae) - what do we know? The Thünen Institute of Forest Genetics, 8pages. DOI: 10.1515/sg-2017-0001.

Millogo AMD. 2014. Etude des caractéristiques morphologiques et de la viabilité des semences de Parkia biglobosa (Jacq.) R. Br. ex G. Don. germoplasme de conservation à long terme à $4{ }^{\circ} \mathrm{C}$. Mémoire de Master, Institut de Développement Rural, Université Polytechnique de BoboDioulasso, 42 p.
Modupe IB. 2014. Parkia biglobosa (African locust bean tree). World Journal of Pharmaceutical research, 3(2): 16721682.

Ntui VO, Uyoh EA, Urah IS, Ogbu U, Okpako EC. 2012. Regeneration of Parkia biglobosa Benth: an important tree species of Africa. Journal of Microbiology and Biotechnology Research, 2(1): 169-177.

Olujobi OJ. 2012. Comparative evaluation of nutritional composition of african locust bean (Parkia biglobosa) fruits from two locations. Nigerian Journal of Basic and Applied Sciences, 20(3): 195-198.

Shao M. 2000. Parkia biglobosa: Changes in resource allocation in Kandiga, Ghana. MSc thesis, Michigan Technical University, Michigan, $106 \mathrm{p}$.

Sina S. 2006. Reproduction et Diversité Génétique chez Parkia biglobosa (Jacq.) G. Don. $\mathrm{PhD}$ thesis, Wageningen University, the Netherlands, $102 \mathrm{p}$.

Sodique FR. 2016. Economics of processed Parkia biglobosa seeds (Daddawa) marketing in Ibadan, Nigeria. Journal of Forests Science and Environment, 1(1): 37-43.

Teklehaimanot Z. 2004. Exploiting the potential of indigenous agroforestry trees: Parkia biglobosa and Vitellaria paradoxa in sub-Saharan Africa. Agroforestry Systems, 61: 207-220. DOI :

https://doi.org/10.1023/b:agfo.00000290 00.22293.d1. 